\title{
Lignes de Kossel observées avec des multicouches périodiques $\mathrm{Fe} / \mathrm{C}$
}

\author{
J.P. Chauvineau, O. Hainaut et F. Bridou \\ Institut d'Optique, URA 14 du CNRS, Centre Universitaire, BP. 147, 91403 Orsay cedex, France
}

\begin{abstract}
Résumé: On met en évidence la diffraction par la multicouche elle-même du rayonnement de fluorescence caractéristique respectivement $\mathrm{X}$ et $\mathrm{X}$ mous des deux éléments constitutifs d'un empilement périodique $\mathrm{Fe} / \mathrm{C}$. Au voisinage des directions d'émergence correspondant à un angle de Bragg du milieu stratifié périodique pour le rayonnement caractéristique de chacun des deux éléments, il apparaît des modulations angulaires de l'intensité de fluorescence - semblables aux lignes de Kossel observées avec les réseaux périodiques tridimensionnels - dont la forme est liée au profil de concentration de l'élément en fonction de la profondeur comme le montre une modélisation basée sur l'utilisation du théorème de réciprocité et le calcul des propriétés optiques des multicouches.
\end{abstract}

\begin{abstract}
The angular distribution of the characteristic X-ray and soft X-ray fluorescence lines of the two elements constituting a periodic Fe/C multilayer is determined experimentally. It is observed that the intensity is strongly modulated in the vicinity of emergence angles corresponding to a Bragg diffraction peak of the periodic stratified medium for each of the two characteristic lines. A modelisation of the intensity distribution is obtained by using the classical theory of optics of thin films and applying the reciprocity theorem.
\end{abstract}

\section{INTRODUCTION.}

Le rayonnement caractéristique émis dans un monocristal par fluorescence d'un élément peut être diffracté par le cristal lui-même; ceci donne lieu à des modulations dans la distribution angulaire de l'intensité du rayonnement de fluorescence divergent issu du cristal et conduit à l'observation d'un diagramme formé de lignes appelées "lignes de Kossel" sur un film plan placé à proximité [1]. Nous voulons montrer dans cette étude qu'un milieu stratifié périodique tel qu'une multicouche destinée à réfléchir le rayonnement $X-U V$, constituée par un empilement périodique de strates alternées de très faible épaisseur peut induire des effets semblables avec le rayonnement de fluorescence de chacun de ses éléments constitutifs. Des effets de l'interférence du rayonnement de fluorescence dans des matériaux stratifiés ont déjà été observés dans des bicouches métalliques [2].

Les multicouches synthétiques périodiques sont maintenant d'un usage bien établi pour la réalisation de composants optiques tels que des miroirs fonctionnant en incidence normale dans le domaine du rayonnement X-UV ou en incidence rasante pour les rayons $X$ [3]. Dans la plupart des cas, elles sont constituées par deux matériaux dont les indices optiques en X-UV sont les plus différents possible pour obtenir un pouvoir réflecteur important; pratiquement, on associe un métal relativement dense comme matériau de "haut indice" à un élément léger $(\mathrm{B}, \mathrm{C}, \mathrm{Si}, \ldots)$ comme matériau de "bas indice". En conséquence les rayonnements de fluorescence caractéristiques de type $\mathrm{K} \alpha$ émis par de telles multicouches se situeront respectivement dans le domaine des rayons $X$ pour le métal et dans celui des X mous pour l'élément léger. 
L'intérêt pratique d'étudier la fluorescence pour faire de l'analyse élémentaire de couches superficielles a été démontré par plusieurs travaux dans lesquels on a mis à profít la variation de la profondeur de pénétration [4] et la modulation d'intensité [5] du rayonnement primaire dans l'échantillon en fonction de l'angle en incidence rasante pour établir des profils de concentration au voisinage de la surface. Notre objectif est de montrer que l'étude de la distribution angulaire du rayonnement de fluorescence des multicouches périodiques peut constituer une méthode de caractérisation non destructive capable de déterminer le profil de concentration en profondeur d'un élément donné, notamment au niveau des interfaces où ce profil influe considérablement sur les performances optiques en X-UV de l'empilement.

\section{DISPOSITIFS EXPERIMENTAUX.}

\subsection{Multicouches.}

Les multicouches $\mathrm{Fe} / \mathrm{C}$ sont préparées par pulvérisation ionique sous atmosphère d'argon $\left(2.10^{-4} \mathrm{mbar}\right)$. L'épaisseur des couches élémentaires est contrôlée par réflectométrie de rayons $X$ mous pendant le dépôt. La période est voisine de $5 \mathrm{~nm}$, les épaisseurs des couches de fer et de carbone étant sensiblement égales. Le nombre de périodes dans l'empilement est de 25. Le substrat - un disque de silicium monocristallin dont la surface polie a une rugosité très faible - est maintenu à la température ambiante pendant le dépôt des couches. L'analyse des multicouches par réflectométrie de rayons $\mathrm{X}$ rasants fait apparaître les pics de Bragg caractéristiques de leur structure périodique dans la direction perpendiculaire au substrat, alors qu'aucune structure particulière n'est observable en diffraction $\mathrm{X}$ à grand angle, indiquant le caractère très désordonné des matériaux à l'échelle atomique dans les couches ultra-minces de fer et de carbone.

\subsection{Fluorescence du fer.}

L'étude expérimentale du rayonnement de fluorescence du fer à $6400 \mathrm{eV}$ (raies Ka1,2) a été faite à l'aide d'un diffractomètre classique équipé d'un tube à anode de cuivre.

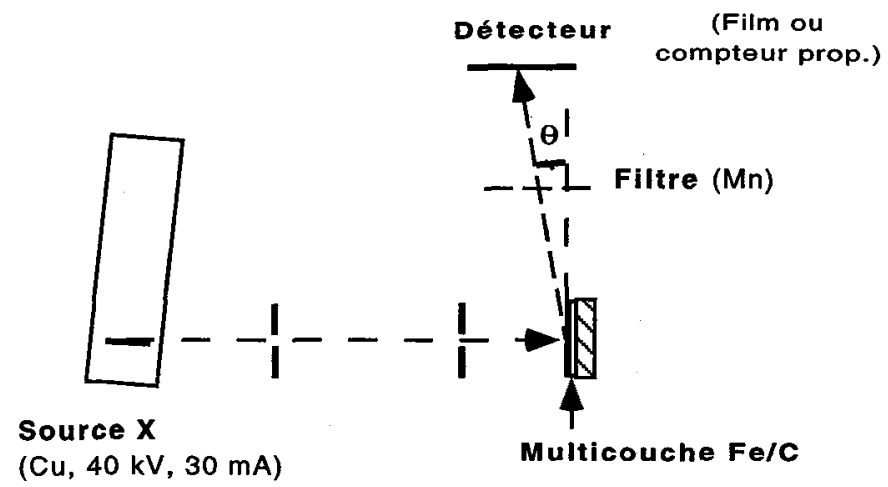

Figure 1: Dispositif expérimental de mesure de l'intensité du rayonnement de fluorescence K $\alpha$ du fer en fonction de l'angle d'émergence rasante $\theta$.

Experimental set up for measurement of the $\mathrm{Fe} \mathrm{K} \alpha$ intensity vs glancing-emergence angle $\theta$.

Pour cette raie du fer, l'angle d'émergence correspondant à la réflexion de Bragg du premier ordre dans l'empilement est voisin de $1^{\circ}$. La multicouche est éclairée sous incidence normale par le rayonnement issu du tube, et la partie du rayonnement de fluorescence émise au voisinage de l'émergence rasante est enregistrée sur un film plan 
perpendiculaire au substrat ou mesurée en fonction de l'angle d'émergence grâce à un compteur proportionnel placé derrière une fente d'analyse. Dans ce dernier cas, un filtre de manganèse ou un monochromateur en graphite peuvent être interposés entre l'échantillon et le détecteur pour réduire l'intensité du rayonnement $K \boldsymbol{\beta}$ du fer par rapport au rayonnement $\mathrm{K} \alpha$ (Figure 1). La durée d'exposition du film est de l'ordre de 4 heures, tandis que le temps de comptage avec le compteur proportionnel est de 100s par point.

\subsection{Fluorescence du carbone.}

La fluorescence du carbone à $277 \mathrm{eV}$ (raie $\mathrm{K} \alpha$ ) a été étudiée sous vide dans une enceinte conçue initialement pour la préparation des multicouches par évaporation thermique. Le canon à électrons à double creuset équipé de cibles en cuivre a été utilisé comme source primaire de rayons $X$; chaque cible bombardée par un faisceau d'électrons de $7 \mathrm{keV}$ et $150 \mathrm{~mA}$ émettait un rayonnement à spectre large constitué essentiellement par le rayonnement de freinage des électrons et les raies $\mathrm{L}$ du cuivre. La détection du rayonnement de fluorescence du carbone était assurée par un compteur proportionnel à fenêtre en polypropylène de $2 \mu \mathrm{m}$ d'épaisseur utilisé pour le contrôle d'épaisseur des couches ultra-minces par réflectométrie de rayons $\mathrm{X}$ mous (Figure 2). Pour pallier le manque de stabilité de la source primaire, on a divisé l'intensité de la raie de fluorescence du carbone par celle du silicium à $1740 \mathrm{eV}$ émise par le substrat et enregistrée simultanément. Malgré la faible valeur du rendement de fluorescence pour le carbone (environ $2.10^{-3}$ ), ce dispositif s'est avéré suffisamment efficace pour obtenir une précision satisfaisante avec des temps de comptage de l'ordre de quelques minutes par point.

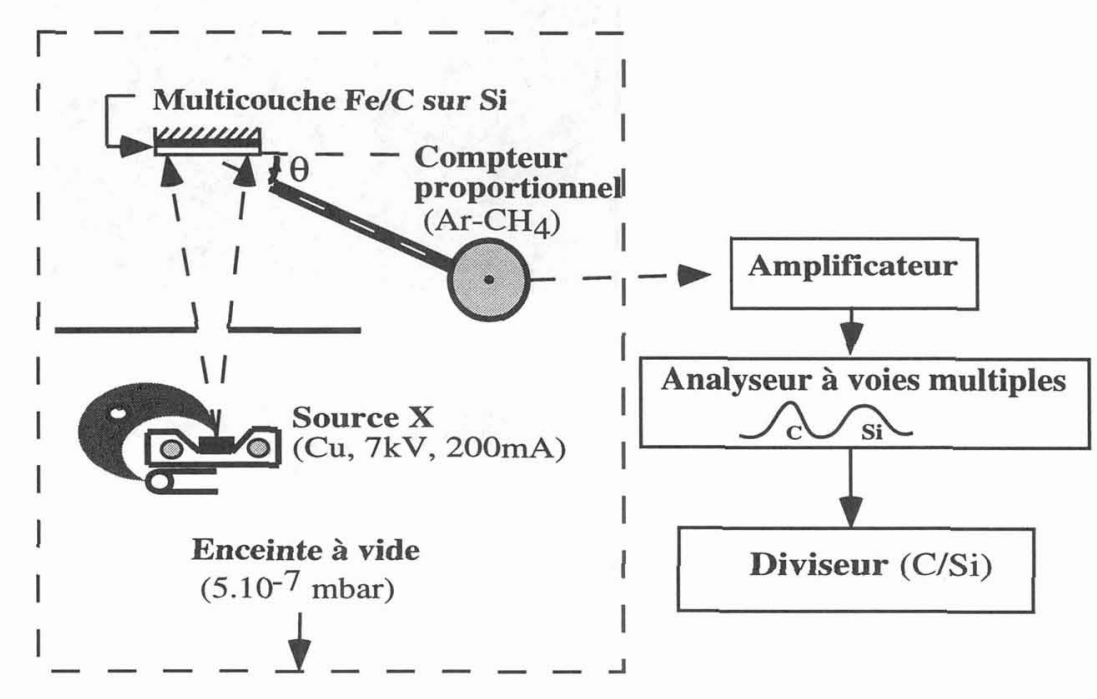

Figure 2: Dispositif expérimental de mesure de l'intensité du rayonnement de fluorescence $\mathrm{K} \alpha \mathrm{du}$ carbone en fonction de l'angle d'émergence $\theta$.

Experimental set up for measurement of the $\mathrm{C} \mathrm{K} \alpha$ intensity vs emergence angle $\theta$. 


\section{RESULTATS.}

On observe bien avec chacun des éléments une modulation de l'intensité du rayonnement de fluorescence en fonction de l'angle d'émergence, localisée au voisinage de l'angle correspondant au pic de Bragg du premier ordre de la multicouche pour les deux longueurs d'onde des raies caractéristiques respectivement du fer et du carbone.

Dans le cas du fer, on distingue sur l'enregistrement photographique de la figure 3a: - une première zone située entre $0^{\circ}$ et $0^{\circ}, 4$ dans laquelle l'intensité est très faible; elle résulte de la réfraction à l'interface multicouche/vide du rayonnement $\mathrm{K} \alpha$ émis par le fer;

- une zone éclairée au-delà de $0^{\circ}, 4$, dans laquelle apparaissent tout d'abord un premier pic large situé immédiatement au-delà de la zone de réflexion quasi-totale, puis entre $1,1^{\circ}$ et $1,2^{\circ}$ une modulation constituée principalement de deux bandes étroites parallèles et juxtaposées, respectivement sombre et claire; cette dernière structure constitue la "ligne de Kossel" due à la diffraction au premier ordre du rayonnement de fluorescence $\mathrm{K} \alpha$ du fer par la multicouche périodique.

Ces structures se retrouvent sur l'enregistrement de l'intensité mesurée sur le diffractomètre avec le compteur proportionnel (figure3b) qui permet un repérage plus précis des angles et élimine les effets de non-linéarité du film. Néanmoins le rapport signal sur bruit est plus faible que pour le film et ne permet plus de résoudre les oscillations secondaires observables sur le film.

a)
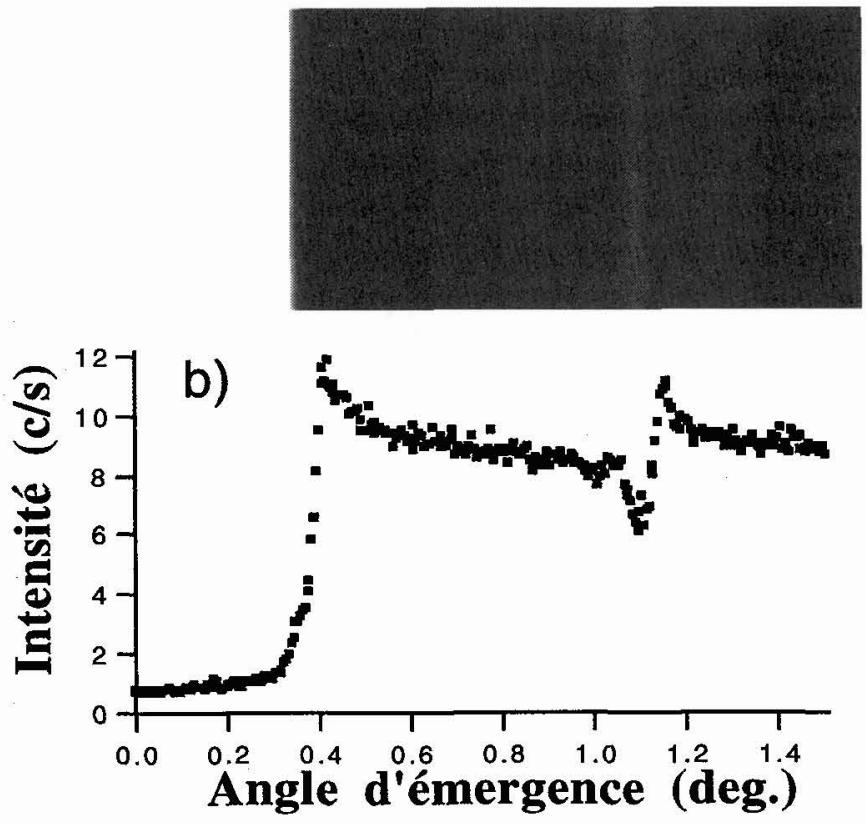

Figure 3: Fluorescence du fer dans une multicouche $\mathrm{Fe} / \mathrm{C}$ périodique enregistrée en émergence rasante a): sur un film photographique;

b): avec un compteur proportionnel sur un goniomètre. 
Pour le carbone, la courbe de variation de l'intensité en fonction de l'angle d'émergence montre une modulation importante située au voisinage d'un angle d'émergence de $29^{\circ}$ correspondant à l'angle de Bragg du premier ordre de la multicouche pour la raie $K \alpha$ du carbone (figure 4). Cette modulation s'apparente à celle qui a été observée dans le cas du fer, avec une inversion dans l'ordre d'apparition des deux bandes qui sont ici respectivement de forte et faible intensité quand l'angle d'émergence augmente. L'incertitude sur la mesure est plus grande que pour le fer en raison du très faible rendement de fluorescence du carbone.

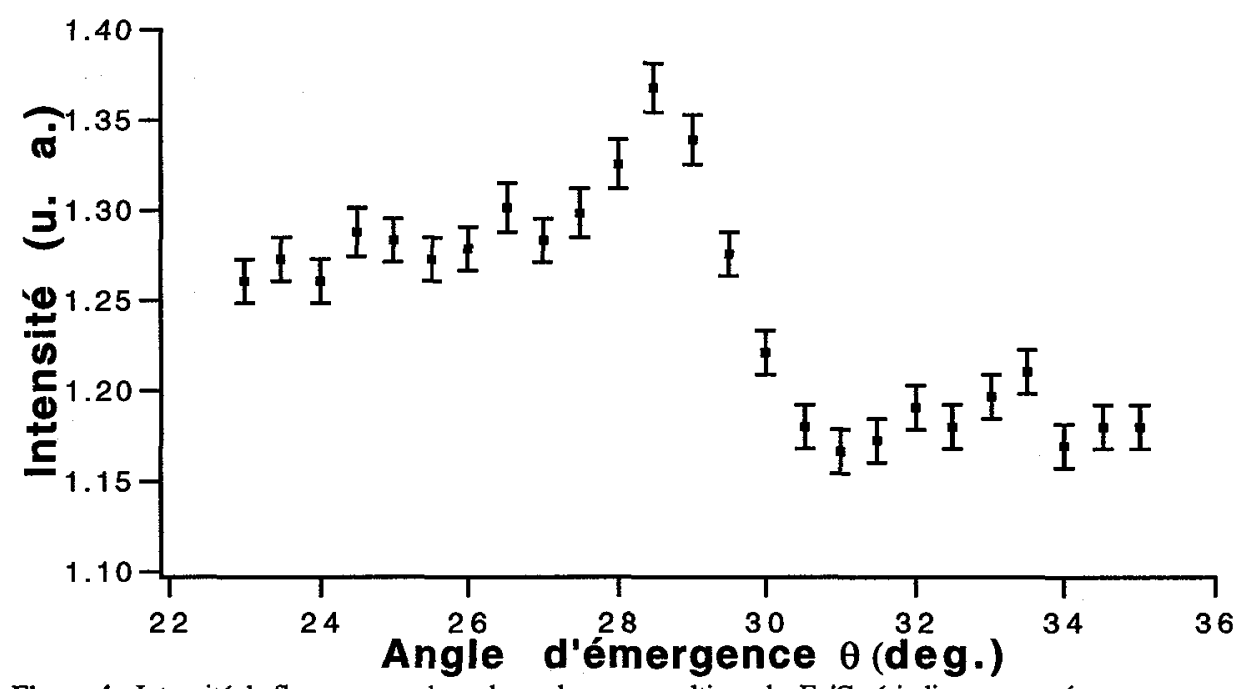

Figure 4 : Intensité de fluorescence du carbone dans une multicouche $\mathrm{Fe} / \mathrm{C}$ périodique, mesurée avec un compteur proportionnel.

\section{MODELISATION.}

Nous avons calculé l'intensité du rayonnement de fluorescence en fonction de l'angle d'émergence en appliquant le théorème de réciprocité [6] selon la méthode utilisée par Laue [7] qui a permis l'interprétation des diagrammes de Kossel. D'après ce théorème, le champ électrique créé à l'entrée du détecteur par la fluorescence d'un atome dans la couche a la même valeur que celui qui serait créé au point où se trouve l'atome par une source ponctuelle identique placée à l'entrée du détecteur. La distance entre l'échantillon et le détecteur étant très grande par rapport à.l'épaisseur des couches, on peut aisément calculer ce dernier champ électrique en tout point de l'empilement en supposant que la source fictive est située à l'infini dans la direction de détection et en utilisant le formalisme classique de calcul des propriétés optiques des couches minces [8]. Comptetenu de la géométrie du système, ce champ ne dépend que de la profondeur dans la multicouche. Il faut ensuite ajouter les contributions de tous les atomes de l'élément considéré, ce qui suppose la connaissance du profil de concentration de cet élément et de l'intensité du rayonnement excitateur en fonction de la profondeur.

Nous montrons sur les figures $5 a$ et $b$ les résultats du calcul de l'intensité de fluorescence du fer et du carbone en fonction de l'angle d'émergence réalisé en supposant d'une part 
que l'intensité du champ excitateur est constante en raison notamment de la faible épaisseur des multicouches par rapport à la profondeur de pénétration du rayonnement primaire et d'autre part que l'empilement est parfaitement périodique, avec des interfaces idéales sans rugosité ni interdiffusion des deux éléments. Les calculs ont été faits pour les deux cas de polarisations " $s$ " et "p", puis en prenant la valeur moyenne.
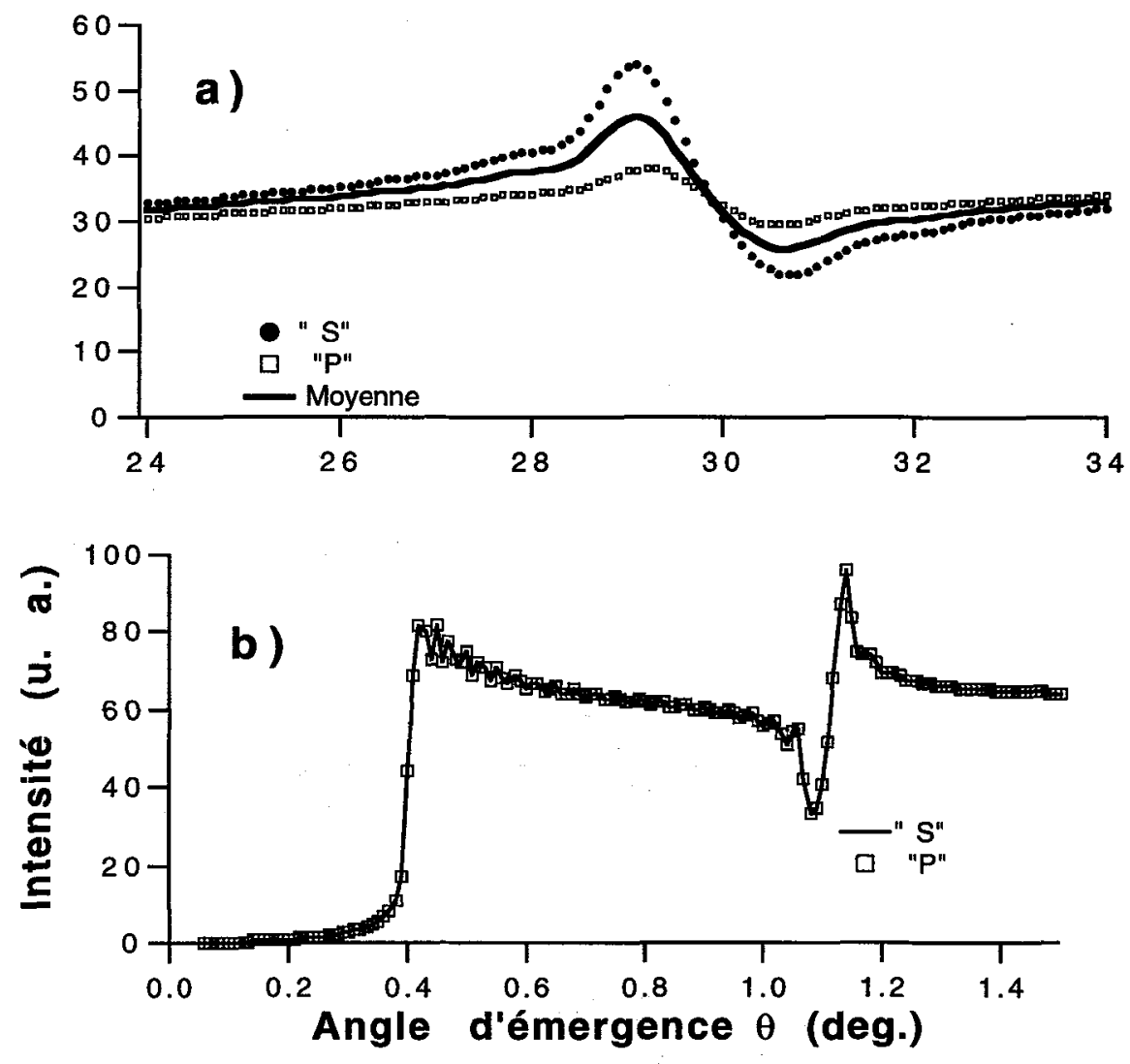

Figure 5: Intensité de fluorescence du carbone (a) et du fer (b) dans une multicouche Fe/C périodique, calculée en utilisant le théorème de réciprocité.

On constate une analogie marquée avec les courbes expérimentales, et notamment la présence de fortes modulations situées d'une part au bord de la zone de réflexion quasitotale pour le fer et d'autre part au voisinage des angles de Bragg du premier ordre pour chacun des deux éléments. Il apparaît également des oscillations de plus faible contraste que l'on peut effectivement observer sur l'enregistrement photographique du rayonnement de fluorescence du fer.

On note que l'effet de la polarisation sur l'intensité n'apparaît pratiquement pas en émergence rasante pour le fer, mais par contre se manifeste assez nettement en $\mathrm{X}$ mous dans le cas du carbone où l'angle d'émergence se rapproche de l'angle de Brewster, proche de $45^{\circ}$ pour les rayons $X$ et $X$ mous. 


\section{CONCLUSION.}

Nous avons mesuré la distribution angulaire du rayonnement de fluorescence $K \alpha$ du fer et du carbone émis par des multicouches périodiques $\mathrm{Fe} / \mathrm{C}$. On observe dans les deux cas une forte modulation de l'intensité émise au voisinage de l'angle d'émergence correspondant à la direction de diffraction de Bragg du premier ordre du rayonnement caractéristique de chaque élément par le milieu stratifié périodique. Sur un film photographique, cette modulation apparaît comme une ligne formée de deux bandes juxtaposées respectivement sombre et claire, semblable aux lignes de Kossel observables sur les diagrammes de fluorescence de monocristaux.

La position et la forme de ces modulations sont bien reproduites par un calcul d'intensité de fluorescence basé sur l'utilisation du théorème de réciprocité et $d u$ formalisme de calcul des propriétés optiques des couches minces.

L'étape suivante de ce travail consiste à tenir compte d'une part de la variation en fonction de la profondeur du champ d'excitation dû au rayonnement primaire renforcé éventuellement par le rayonnement de fluorescence du substrat en silicium dans le cas du carbone, et d'autre part des défauts des multicouches en ajustant le profil de concentration des éléments aux interfaces pour simuler quantitativement les courbes expérimentales.

Dans la pratique, on envisage aussi d'irradier l'échantillon avec un rayonnement monochromatique en incidence rasante au voisinage d'un angle de Bragg pour moduler l'intensité du champ électrique en fonction de la profondeur et par conséquent amplifier ou atténuer à volonté l'émission de certaines zones des multicouches [5].

\section{Références.}

[1] W. Kossel, V. Loeck, H. Voges; Zeit. f. Physik; 94; 1935; p. 139.

[2] Noma T., Iida A., Sakurai K., Phys. Rev. B, 48,(1993), 17524-17526.

[3] Spiller E., Soft X-ray Optics, (SPIE Opt. Eng. Press, Bellingham, Washington, 1994) pour une bibliographie détaillée sur ce sujet.

[4] Brunel M., de Bergevin F., Acta Cryst., A42, (1986), 299-309.

[5] de Boer D. K. G., Phys. Rev. B, 44, (1991), 498-511.

[6]. H.A. Lorentz; Proc. Amsterdam Acad.; 8; 1905; p.401.

[7]. M. v. Laue; Ann. der Physik; 23; 1935, p.705.

[8]. Berning P. H., Physics of thin films, 1, (G. Hass, Academic Press, 1963), 69. 